\title{
Um Experimento para Medir Velocidades como Instrumento Motivador no Aprendizado de Conceitos da Ótica Física
}

\author{
M. Müller, J. L. Fabris, R. Falate, E. Cação Jr. \\ Centro Federal de Educação Tecnológica do Paraná \\ Av. 7 de setembro, 3165, 80230-901, Curitiba, PR
}

An Experiment for Measurements of Speed as a Motivation Tool for Learning some Optical Physics Concepts

Recebido em 25 de novembro, 2002. Aceito em 28 de maio, 2003.

\begin{abstract}
Neste artigo é descrito um arranjo experimental que forma a base para operação dos Velocímetros Laser. O arranjo é usado na medição da velocidade de um sistema físico bem conhecido. A utilização deste arranjo experimental possibilita ao aluno a compreensão dos fenômenos de interferência, difração e espalhamento da luz, com a utilização de uma técnica ótica com inúmeras aplicações no campo de medidas de velocidades de partículas. Como sistema físico a ser analisado é utilizado um pêndulo simples, cuja velocidade pode ser facilmente calculada, permitindo sua comparação com os resultados experimentais.

This article describes an experimental apparatus that forms the basis for operation of Laser Velocimeters. The setup is used to measure the velocity of a well-known physical system. The use of this experimental apparatus allows the students to understand the effects of interference, diffraction and scattering of light, by means of an optical measurement technique with several applications in particles velocities measurements. A simple pendulum is the physical system chosen to be analyzed, once its velocity can be easily calculated and the theoretical predictions compared with the experimental values.
\end{abstract}

\section{Introdução}

Com a aceitação da teoria de Fresnel para as ondas de luz, a compreensão dos fenômenos de interferência e difração, resultantes do comportamento ondulatório da luz, tornou possível o desenvolvimento de um grande número de componentes e dispositivos óticos amplamente usados na indústria, medicina e comunicações [1,2,3]. Além disso, foram desenvolvidas inúmeras técnicas óticas de medidas que têm sido aplicadas com sucesso em vários campos da pesquisa [4]. Entre estas encontra-se a Velocimetria Laser que permite a medida de velocidades de sistemas físicos onde um método de medida não invasivo é requerido. Numa das configurações possíveis desta técnica [5], a medida da velocidade das partículas de um meio é realizada usando as franjas do padrão de interferência formado no cruzamento de dois feixes laser. Este padrão de interferência é focalizado dentro de um fluido cuja velocidade se deseja medir. Pequenas partículas contidas dentro do fluido que cruzam este padrão espalham luz modulada com uma freqüência proporcional a velocidade do fluido. Apesar desta configuração não detectar o desvio Doppler da freqüência da luz, ela é a base de velocímetros laser comerciais. Na Velocimetria Laser não existe contato físico com o corpo de prova, o que torna a técnica útil na análise da velocidade de fluidos corrosivos ou venenosos. Como o feixe laser pode ser focalizado com precisão em um ponto muito pequeno, este método também permite a medição da velocidade de um fluido ponto a ponto, possibilitando o levantamento do perfil de velocidades do escoamento e a compreensão da cinemática dos fluidos.

Uma das grandes dificuldades encontradas pelos professores que ministram aulas de física básica é a falta de motivação dos alunos para estudar conceitos físicos. Isto ocorre principalmente devido a grande dificuldade que os alunos encontram em relacionar conceitos básicos com aplicações tecnológicas da sua área de interesse. Sendo assim, buscamos, com este trabalho, minimizar esta dificuldade quando estão sendo trabalhados assuntos relacionados com ótica física. Os principais objetivos são atrair o interesse do aluno pelo estudo da ótica física mediante uma correlação entre os assuntos tratados normalmente em sala de aula e uma aplicação tecnológica, e contribuir para o enriquecimento da sua formação por meio do contato com uma tecnologia ótica. O sistema apresentado usa os princípios da velocimetria laser numa montagem experimental que pode ser realizada com certa facilidade. O sistema é empregado na medição da velocidade máxima de um pêndulo simples, o que permite uma rápida verificação dos valores medidos por meio da comparação com valores previamente calculados.

\section{Teoria}

Quando a luz proveniente de um laser, separada em dois feixes coerentes de mesma amplitude e polarização é no- 
vamente superposta, são observadas na região de interseção franjas claras e escuras resultantes da interferência da luz [6]. A separação $d$ entre as franjas claras consecutivas desse padrão pode ser controlada, uma vez que esta depende do comprimento de onda da luz laser usada, e também do ângulo de cruzamento $\psi$ entre os feixes. O desenho da Fig. 1 mostra o cruzamento entre dois feixes laser de diâmetros $b$, e representa as franjas claras e escuras do padrão de interferência resultante na região de cruzamento. Quando uma partícula com dimensões inferiores a separação entre as franjas atravessa o padrão, ocorrerá um espalhamento de luz sempre que esta passar nas regiões de franjas claras. Um detector posicionado adequadamente de forma a captar a luz espalhada pela partícula durante a sua trajetória receberá uma intensidade luminosa variável. A freqüência do sinal detectado é proporcional a taxa com que a partícula cruza as franjas do padrão de interferência, e desta forma, conhecendo-se a separação entre as franjas, a velocidade da partícula pode ser facilmente calculada.

Dependendo das condições nas quais o experimento está sendo realizado, a separação dentre as franjas do padrão pode ser calculada usando o valor medido do ângulo $\psi$ entre os feixes, ou medida diretamente no padrão. Quando a técnica é usada para medir a velocidade de um fluido em uma canalização, é utilizado um padrão onde o espaçamento $d$ entre as franjas é da ordem do pequeno diâmetro das partículas espalhadoras de luz. Estas partículas podem tanto estar presentes na própria composição do fluido quanto ser intencionalmente adicionadas a ele. O padrão é obtido por meio do cruzamento de feixes focalizados de diâmetro bastante reduzido num ângulo $\psi$ que pode ser controlado. Assim, têm-se a formação do padrão de interferência em regiões pequenas, possibilitando um levantamento das velocidades em diferentes posições dentro da tubulação. Estas características, aliadas ao fato de que as medidas são realizadas por meio do cruzamento dos feixes dentro da canalização, deixam claro que neste caso é mais adequado calcular o espaçamento entre as franjas do que medí-lo diretamente. Este cálculo é realizado usando a diferença de percurso entre os dois feixes que se cruzam em diferentes pontos da região de superposição. Para isto, vamos considerar um ponto $P$ na Fig. 1, deslocado de uma distância $d$ em relação ao eixo central do cruzamento. Os dois feixes percorrem caminhos óticos diferentes até chegar a esse ponto. A diferença de caminho ótico $\Delta r$ entre os dois feixes corresponde a diferença entre as distâncias $r_{1}$ e $r_{2}$ indicadas na figura é dada por

$$
\Delta r=r_{2}-r_{1} .
$$

Aplicando o teorema de Pitágoras aos triângulos ABP e APD da figura são encontradas as relações:

$$
\begin{aligned}
& r_{2}^{2}=a^{2}+d^{2} \\
& r_{2}^{2}=b^{2}+r_{1}^{2}
\end{aligned}
$$

em que $a=r_{2} \cos \left(\frac{\Psi}{2}\right), d=r_{2} \operatorname{sen}\left(\frac{\Psi}{2}\right)$, e $b=$ $r_{2} \cos \left(\frac{\pi}{2}-\Psi\right)$.

Estas relações fornecem a dependência entre as distâncias $r_{1}$ e $r_{2}$ com o ângulo de cruzamento $\psi$, na forma

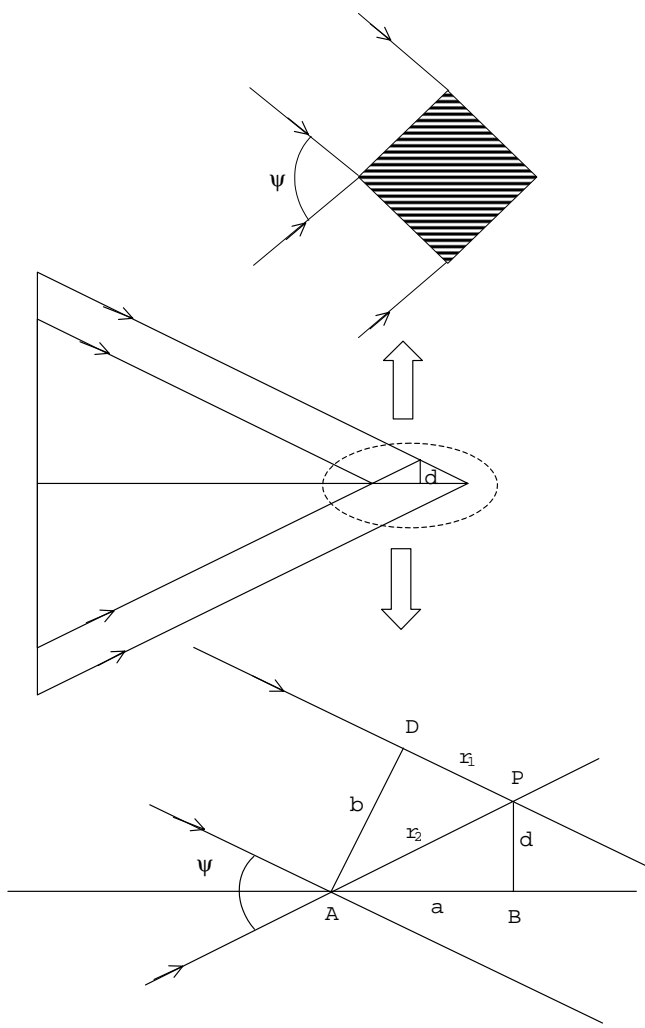

Figura 1. Diagrama mostrando a região de intersecção entre dois feixes laser de diâmetros $b$ num ângulo de cruzamento $\psi$. Ilustração das franjas claras e escuras do padrão de interferência resultante na região de cruzamento entre dois feixes laser (detalhe superior). Ampliação da região de cruzamento, mostrando um ponto $P$ deslocado de $d$ em relação ao eixo central do cruzamento (detalhe inferior).

$$
r_{1}=r_{2} \cos \Psi
$$

Assim, a diferença de percurso pode ser escrita em função do ângulo de cruzamento e da posição com relação ao eixo central na forma

$$
\Delta r=2 d \operatorname{sen} \frac{\Psi}{2}
$$

A intensidade resultante da interferência de duas ondas de igual frequiência é

$$
I=4 I_{0} \cos ^{2}\left(\frac{\Phi}{2}\right)
$$

em que $\phi$ é a diferença de fase entre as ondas. Essa diferença de fase irá depender da diferença de percurso entre as duas ondas e produzirá uma interferência construtiva sempre que $\Delta r$ for igual a um número inteiro de comprimentos de onda. Assim, franjas claras consecutivas possuem uma separação dada por

$$
d=\frac{\lambda}{2 \operatorname{sen}(\Psi / 2)} .
$$


A luz espalhada pela partícula que atravessa este padrão de franjas de interferência com velocidade $v$ é captada por um detector. O sinal detectado possui uma freqüência que depende da velocidade da partícula e da separação entre as franjas claras dada por

$$
f=\frac{v}{d}
$$

Medindo essa freqüência e o ângulo de cruzamento entre os feixes é possível obter a velocidade da partícula através de

$$
v=\frac{f \lambda}{2 \operatorname{sen}(\Psi / 2)}
$$

Para que a faixa de variação do sinal elétrico na saída do detector seja grande, permitindo uma determinação adequada dos pontos de máximo e mínimo, a dimensão das partículas deve ser inferior à separação entre as franjas. Esta condição pode ser alcançada ajustando o ângulo $\psi$ de interseção dos feixes. Se as medidas de velocidade a serem realizadas possibilitam o uso de partículas espalhadoras de maior diâmetro, a separação entre as franjas do padrão de interferência também deve ser maior e não há a necessidade de focalização dos feixes. Assim, tem-se um padrão de interferência mais extenso e a separação $d$ entre as franjas pode ser medida diretamente deste padrão. Isto é conseguido efetuando uma varredura da distribuição de intensidade luminosa do padrão por meio do deslocamento, perpendicular às franjas, de um detector montado sobre um estágio translacionador (Fig. 2). A análise dos sinais captados pelo detector em diferentes pontos da sua trajetória fornece facilmente a separação $d$ entre as franjas. É importante notar que a freqüência do sinal medido somente fornecerá o valor real da velocidade da partícula, se esta atravessar a região de interferência numa trajetória perpendicular as franjas.

\section{Pêndulo}

Sabemos que o deslocamento linear $s$ do pêndulo varia harmonicamente no tempo e é dado pela expressão:

$$
s=s_{m} \cos (\omega t+\phi)
$$

em que $s_{m}$ é a amplitude máxima do movimento e $\omega=$ $\sqrt{g / L}$ é a freqüência natural de oscilação do pêndulo que depende do comprimento $L$ do mesmo e da aceleração da gravidade $g$.

A velocidade do pêndulo em cada ponto da trajetória será dada por

$$
v=s_{m} \omega \operatorname{sen}(\omega t+\phi) .
$$

No ponto mais baixo da trajetória o pêndulo adquire a sua velocidade máxima

$$
v_{m}=s_{m} \omega .
$$

Um ponto qualquer do fio a uma distância $y$ do ponto de sustentação terá uma velocidade máxima $v_{m}^{\prime}$ igual a

$$
v_{m}^{\prime}=s_{m} \sqrt{\frac{g y^{2}}{L^{3}}} .
$$

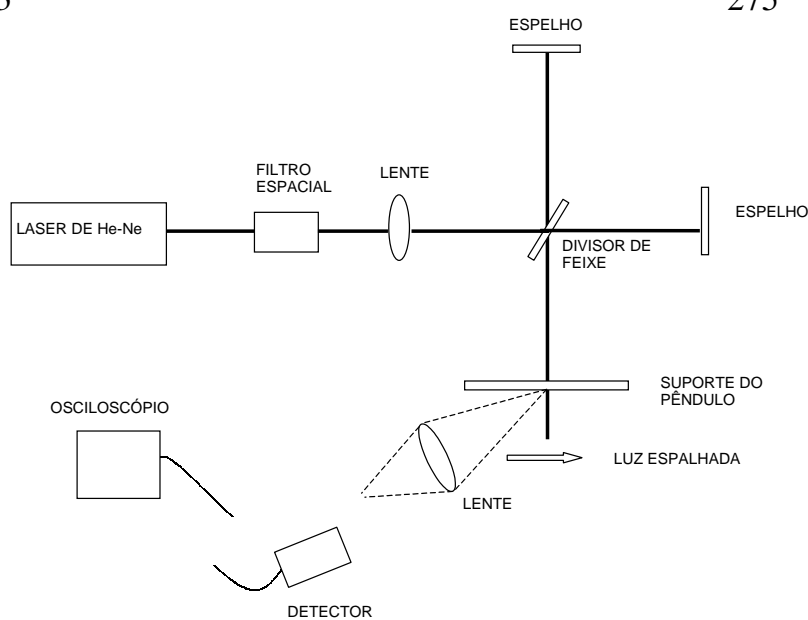

Figura 2. Arranjo experimental do velocímetro usado nas medidas de velocidade do fio de um pêndulo.

\section{Montagem experimental e proce- dimentos}

A seguir é descrita a montagem experimental desenvolvida e a comprovação do seu desempenho por meio da medida da velocidade máxima de passagem do fio de um pêndulo simples na região do padrão de interferência.

$\mathrm{O}$ arranjo experimental do velocímetro está ilustrado na Fig. 2, e consiste basicamente de dois feixes laser coerentes, de mesma amplitude e polarização, que se cruzam formando um ângulo $\psi$. Na região de interseção aparecem planos claros e escuros correspondentes às franjas do padrão de interferência. Usamos como pêndulo uma esfera metálica suspensa por um fio de poliester com diâmetro de $0,3 \mathrm{~mm}$. Usamos um pêndulo com comprimento total de 59 $\mathrm{cm}$ sendo medida a velocidade de um ponto do fio posicionado a aproximadamente $45,5 \mathrm{~cm}$ do eixo de rotação. A amplitude máxima de oscilação do pêndulo em cada medida foi obtida para um deslocamento angular inicial em torno de $7^{\circ}$.

Antes do início das medidas de freqüência foi feita uma varredura do padrão de interferência na posição do pêndulo. Esta varredura tem como objetivo medir a separação $d$ e permitir o seu ajuste com relação ao diâmetro do fio do pêndulo medido por difração. O ângulo de cruzamento deve ser escolhido para que forneça uma separação entre as franjas do padrão de interferência maior do que o diâmetro do fio do pêndulo. Dessa forma é possível garantir uma variação de intensidade luminosa espalhada que pode ser facilmente medida pelo sistema de detecção. Como o fio do pêndulo tem um diâmetro considerável, precisamos expandir os feixes do laser para que possamos obter um padrão de interferência com uma separação adequada entre as franjas e um número de quatro franjas claras a serem cruzadas pelo fio. Assim foi possível medir a modulação da intensidade luminosa espalhada durante a passagem do pêndulo. Este procedimento 
tornou difícil a medição do ângulo de cruzamento e portanto nesta situação foi mais fácil medir diretamente no padrão a separação entre as franjas. Esta medida foi realizada com um detetor OPT101 da Burr-Brown varrendo o padrão de interferência por meio do movimento de um estágio translacionador linear controlado por um motor de passo [7]. No nosso caso, um software é responsável pelo movimento do translacionador e pela aquisição dos dados provenientes do detector. No entanto, tal sistema pode ser substituído por um parafuso micrométrico e a varredura pode ser feita manualmente. Usamos uma fenda estreita feita com duas lâminas de barbear em frente à janela do detector para diminuir a área atingida pela luz e possibilitar a medição das variações de intensidade do padrão. Após o levantamento da separação entre as franjas foi realizada uma série de cinco medidas de freqüência para a primeira passagem do fio do pêndulo através do padrão de interferência. O valor da velocidade do fio foi obtido substituindo os valores medidos de freqüência e separação entre as franjas na equação (7). Este valor foi posteriormente comparado com o valor obtido usando os parâmetros característicos do pêndulo nas equações do movimento do mesmo. Para coletar somente a luz espalhada pelo fio do pêndulo, o detetor foi distanciado do padrão, e uma lente foi usada para focalizar sobre a sua janela a intensidade espalhada lateralmente pelo fio. Para garantir que a trajetória do fio na região das franjas fosse a mais próxima possível de uma trajetória retilínea, usamos um pêndulo longo com $59 \mathrm{~cm}$ de comprimento, e uma máscara contendo uma fenda posicionada na frente das franjas. Tal fenda é responsável pela seleção de uma fração do padrão de interferência, e as suas dimensões foram escolhidas de tal forma que fosse possível registrar a freqüência da passagem do fio através do padrão, sem no entanto introduzir erros muito grandes nos valores medidos causados pela variação da velocidade linear do pêndulo ao longo da sua trajetória. O pêndulo e a máscara foram posicionados de tal forma que a região das franjas coincidisse com a posição da sua velocidade máxima, ou seja, com a região onde o fio do pêndulo fica aproximadamente na vertical.

A montagem do sistema experimental utiliza um laser de He-Ne com potência de $20 \mathrm{~mW}$ e razão de polarização de 1:500. Antes de ser dividido, o feixe do laser passa por um filtro espacial composto por uma lente de 40x com distância focal de 4,3 mm e um orifício circular com diâmetro de $10 \mu \mathrm{m}$. Uma lente com $5 \mathrm{~cm}$ de distância focal torna o feixe paralelo antes de ser separado em dois outros feixes pelo divisor de feixe. Os dois espelhos planos posicionados de maneira a compor os braços do interferômetro, podem ser ajustados para permitir a visualização das franjas e controlar o ângulo de cruzamento dos feixes. Para evitar o borramento do padrão de interferência, todo o sistema é montado sobre uma bancada livre de vibrações externas. Pode-se conseguir um bom isolamento apoiando o tampo da mesa sobre cama- ras de ar.

A medição no osciloscópio da freqüência do sinal detectado, juntamente com o uso da distância medida entre as franjas permite determinar experimentalmente a velocidade $v_{m}^{\prime}$ do ponto $y$ do pêndulo.

Usando as expressões do movimento harmônico simples [8], a velocidade máxima do pêndulo pode ser facilmente calculada pela equação (12) e comparada com as medidas realizadas.

\section{Resultados e conclusões}

Os resultados fornecidos pelo sistema quando aplicado à medida da velocidade do pêndulo são apresentados nas Figs. 3 e 4. A Fig. 3 mostra o resultado experimental do levantamento das intensidades do padrão de interferência na região de passagem do pêndulo, feito com o detector preso ao estágio translacionador. A linha sólida representa o ajuste de uma função cosseno ao quadrado modulada por uma função gaussiana aos dados experimentais. A função cosseno ao quadrado fornece a intensidade das franjas do padrão de interferência (eq. 5), enquanto que a função gaussiana descreve o perfil da intensidade da secção transversal dos feixes laser que modula este padrão. Este ajuste permite medir diretamente o valor da separação entre as franjas. Também é apresentado no detalhe da Fig. 3 o resultado da transformada de Fourier aplicada aos dados experimentais, o que também permite determinar o espaçamento entre as franjas. Para ambas as técnicas de análise, o valor encontrado foi de 2,6 mm. Na Fig. 4, é mostrado um gráfico típico de intensidade de luz espalhada pelo fio do pêndulo em função do tempo, durante uma passagem deste pela região do padrão. Uma freqüência de modulação do sinal de $75,6 \mathrm{~Hz}$ foi obtida aplicando a transformada de Fourier aos pontos experimentais apresentados neste gráfico. Para os resultados apresentados nas Figs. 3 e 4, o valor de velocidade calculado foi de $0,20 \mathrm{~m} / \mathrm{s}$. Este valor apresenta uma discrepância de $10 \%$ em relação ao valor calculado de $0,22 \mathrm{~m} / \mathrm{s}$ com o auxílio da expressão (11) na qual foram usados os parâmetros conhecidos do pêndulo. Esta pequena discrepância entre os dois valores se justifica devido aos erros propagados correspondentes a variações na amplitude máxima de lançamento, e as várias grandezas que necessitam ser medidas, entre elas o comprimento total $L$ do pêndulo e a posição $y$ do ponto do fio cuja velocidade é estimada. Além disso, a velocidade medida corresponde a um valor médio de velocidades devido ao tamanho da máscara, enquanto que o valor calculado usando a equação (11) corresponde a velocidade máxima. A facilidade no processo de medição e a pequena discrepância entre os valores de velocidades fornecidos pela medida e pelo cálculo usando as equações de movimento, demonstram a viabilidade do uso de tal sistema em demonstrações didáticas. 


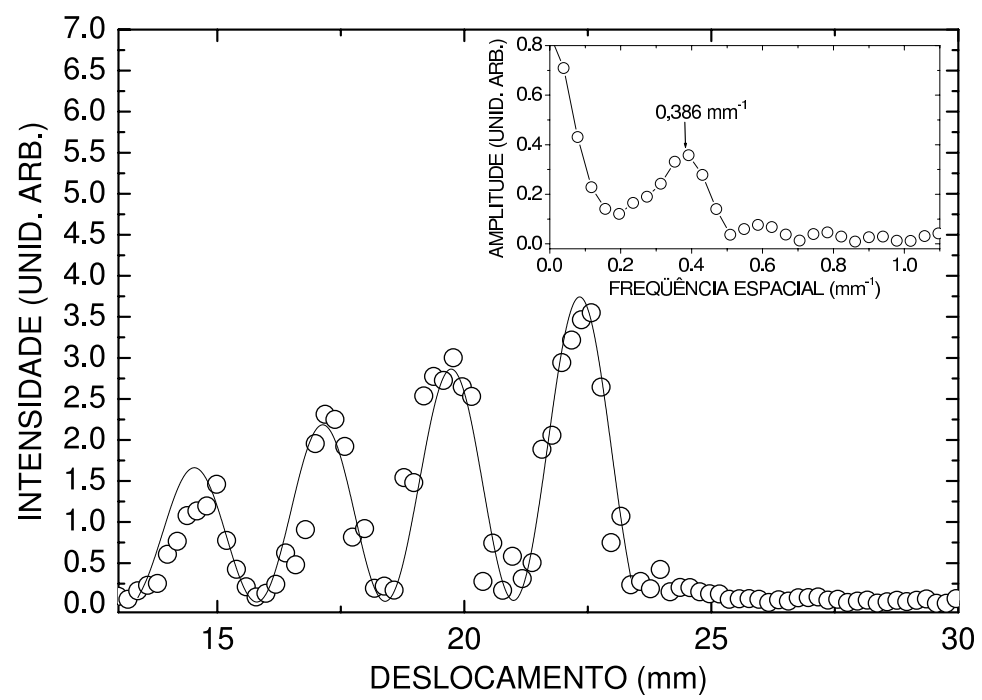

Figura 3. Pontos experimentais do levantamento das intensidades do padrão de interferência na região de passagem do pêndulo, ajustados por uma função cosseno ao quadrado modulada por uma gaussiana. No detalhe é apresentado o resultado da aplicação da transformada de Fourier aos dados experimentais (a linha sólida é apenas para uma melhor visualização).

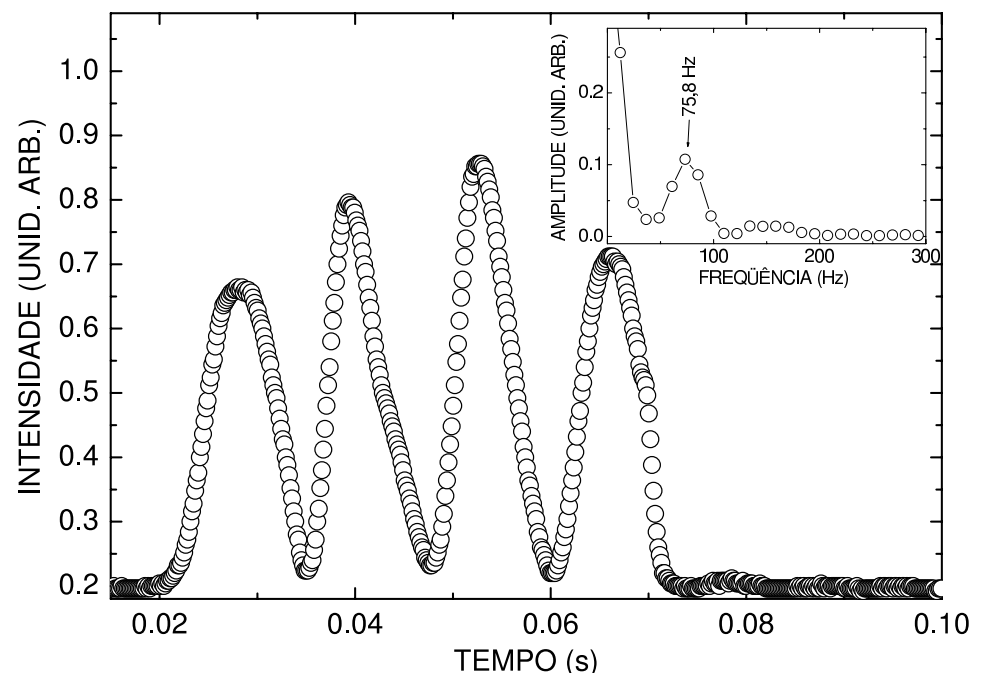

Figura 4. Pontos experimentais do levantamento da intensidade de luz espalhada pelo fio do pêndulo em função do tempo, durante uma passagem deste pela região do padrão. No detalhe encontra-se o resultado da aplicação da transformada de Fourier aos dados experimentais (a linha sólida é apenas para uma melhor visualização).

\section{Referências}

[1] A. Othonos, "Fiber Bragg Gratings", Rev. Sci. Instrum. , 68, 12, 4309- 4341(1997).

[2] T. G. Giallorenzi, J. A. Bucaro, A. Dandridge, G.H. Sigel Jr., J.H Cole, S.C. Rashleigh, R.G. Priest, "Optical Fiber Sensor Technology”, IEEE Journal of Quantum Electronics, QE-18, 4, 626-664 (1982).

[3] H. M. Muncheryan, Laser and Optoelectronic Engineering, Hemisphere Publishing Corporation, (1991).
[4] H. Maillet, O laser- Princípios e técnicas de aplicação, Editora Manole, São Paulo, (1987).

[5] J. F. Ready, Industrial Applications of Lasers, Academic Press, (1978).

[6] Paul A. Tipler, Física, LTC- Livros Técnicos e Científicos LTDA, $4^{a}$ edição, 4, (2000).

[7] J. L. Fabris, R. E. de Góes, R. Falate, M. Müller, Revista de Física Aplicada e Instrumentação, 14, 2, 38-44 (junho 1999).

[8] Paul A. Tipler, Física, LTC- Livros Técnicos e Científicos LTDA, $4^{a}$ edição, 1, (2000). 